\title{
Size and temperature effects on wetting in supercooled vacuum condensates
}

\author{
S.V. Dukarov * \\ Department of Physics, Kharkov State University, 4 Svobody Sq., Kharkov, 310077, Ukraine
}

Received 21 July 1997; accepted 4 December 1997

\begin{abstract}
The measured data of studying the temperature dependence of wetting angles of amorphous carbon films by island tin and indium condensates are presented in the broad temperature range $(350-850 \mathrm{~K})$ encompassing the range of the supercooled state of metals together with the size dependence of wetting in the $\mathrm{Sn} / \mathrm{C}$ at $400 \mathrm{~K}$. Considerable decrease of the wetting angle is observed with the liquid metal supercooling increasing, the fact being probably associated with the decrease of the interphase energy of the drop-substrate boundary. The size effect is observed for the supercooled tin microdrops $(\Delta T=105 \mathrm{~K})$ with the radii $R<30 \mathrm{~nm}$ manifesting itself in the improvement of wetting with the decrease of the microdrop size. (C) 1998 Elsevier Science S.A. All rights reserved.
\end{abstract}

Keywords: Wetting; Amorphous carbon films; Supercooled vacuum condensates

\section{Introduction}

Studies of supercooled state of metals are undoubtedly of interest for understanding melt crystallization kinetics, as well as for solving practical problems, related to the improvement of microstructure and properties of stable and metastable phases. Research into the island films crystallization has established, that supercooling $\Delta T$ depends essentially on the material of a substrate, onto which condensation has been made. The contact angle $\theta$ between the substrate and a liquid droplet of the condensing metal is the parameter determining this dependence [1-3]. In papers [1-3] for the analysis of results on supercooling, one has used the value of angle $\theta$, appropriate to wetting a substrate by a massive drop at the temperature a little above its melting point $T_{\mathrm{s}}$. However, the crystallization of a condensate depending on a material of a substrate is observed considerably below the metal melting point in the bulk as well as under a small supercooling. It proceeds with the participation of small particles (nuclei), for which the surface energy, and consequently, the wetting angle can considerably differ from the values associated with bulk materials. Recently [4-7], for a number of contact systems $(\mathrm{Sn} / \mathrm{C}, \mathrm{In} / \mathrm{C}, \mathrm{Bi} / \mathrm{C}, \mathrm{Pb} / \mathrm{C}, \mathrm{Au} / \mathrm{C}, \mathrm{Pb} / \mathrm{Si})$, the wetting of amorphous neutral substrates by liquid metals

\footnotetext{
*Corresponding author. e-mail: sergei.v.dukarov@univer.kharkov.ua.
}

has been shown to improve with the sizes of microdrops reducing. This effect has been investigated for temperatures, exceeding the melting point of a metal, and it has been shown [4-7] to be a consequence of the reduction of the surface energy of metal drops $\sigma_{1}$ and the interphase energy of a drop-substrate interface $\sigma_{\mathrm{sl}}$.

Therefore, for the description of island films crystallization on a substrate, it is expedient to study the size dependence of a wetting angle under deep supercooling and its temperature dependence in a broad range of temperatures, including a region of the melt supercooled state.

This paper reports the results of investigating the wetting size dependence in supercooled condensates of tin on a carbon substrate at $T=400 \mathrm{~K}$ and the dependence of wetting on temperature (in the range $350-850 \mathrm{~K}$ ) for tin and indium films, condensed by the vapour-liquid mechanism on carbon substrates. The choice of the systems specified is due to that $\mathrm{Sn}$ and In are inert in relation to carbon in the broad range of temperatures that allows to exclude effects connected with mutual solubility and with the formation of new compounds at the interface.

\section{Technique of experiment}

Preparation of samples for studying the size dependence of wetting has been made by Sn evaporation and condensa- 
tion in vacuum $10^{-3}-10^{-4} \mathrm{~Pa}$ on freshly deposited carbon films. To obtain the supercooled particles, the substrate temperature has been kept by approximately $100 \mathrm{~K}$ below $T_{\mathrm{s}}$. Wetting angles have been measured on micrographs of particle profiles on coiled sections of the carbon film (the convolution method $[8,9])$.

To study the temperature dependence of wetting, a technique has been developed permitting in a single experiment to obtain the $\theta(T)$ dependence in the broad temperature range from the maximum supercooling temperature on the substrate chosen to the critical temperature of condensation.
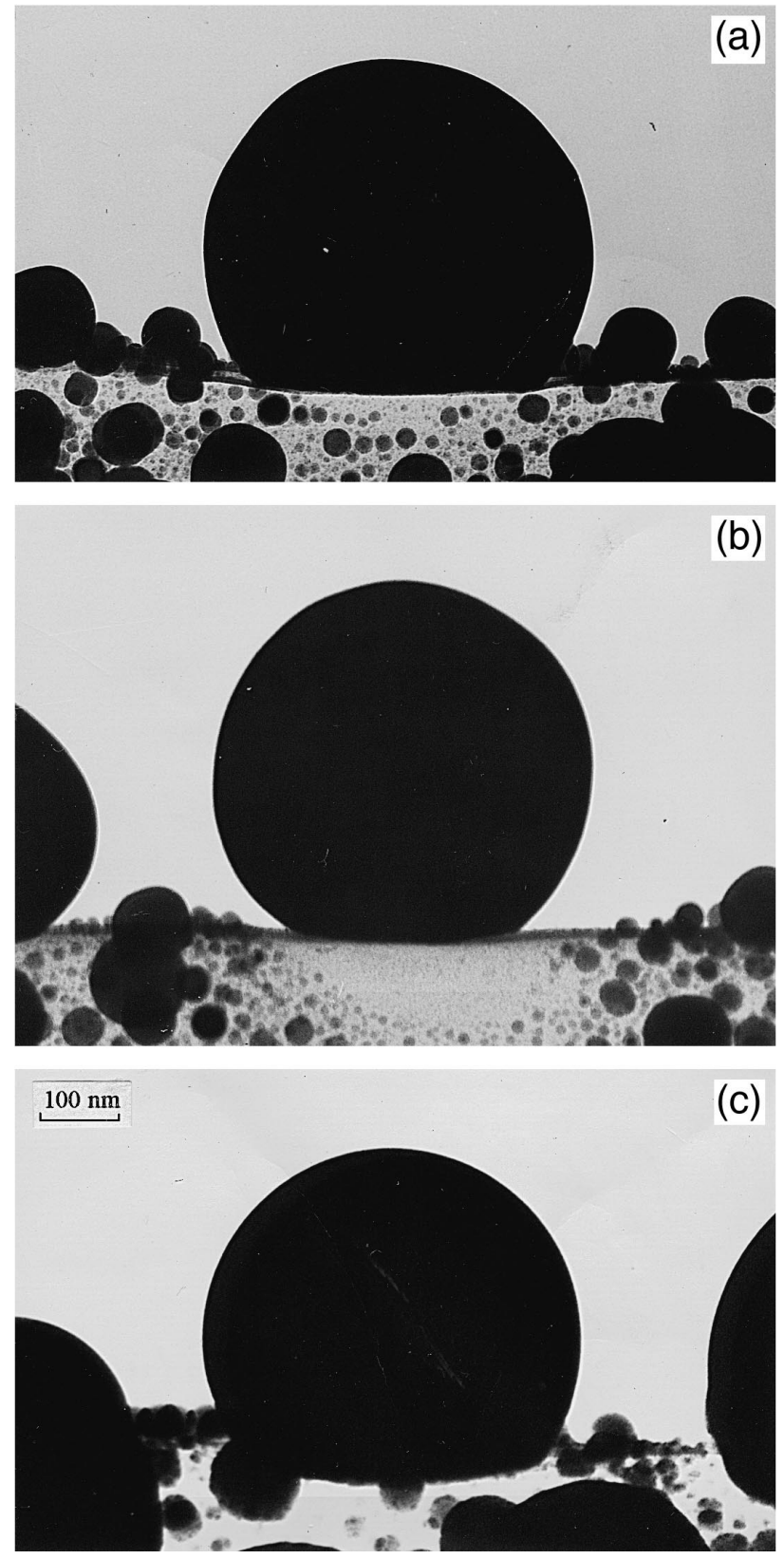

Fig. 1. Micrographs of tin drops deposited on carbon substrates at $400 \mathrm{~K}$ (a), $570 \mathrm{~K}(\mathrm{~b})$ and $730 \mathrm{~K}$ (c).
The samples have been prepared as follows. A film of the metal under study has been deposited by condensation through a liquid phase in vacuum $10^{-4} \mathrm{~Pa}$ on a substrate with the temperature gradient. Amorphous carbon films have been used as substrates, the films being deposited on the $\mathrm{NaCl}$ single crystal cleavages or polished $\mathrm{Al}_{2} \mathrm{O}_{3}$ plates directly before the metal condensation. The $\mathrm{NaCl}$ or $\mathrm{Al}_{2} \mathrm{O}_{3}$ plates have been fixed in a substrate stainless steel $\Omega$ shaped holder. One end of it has been heated to 500-900 $\mathrm{K}$ and another one has been kept at 200-300 K. Thus, the 1-3 $\mathrm{K} / \mathrm{mm}$ temperature gradient has been established on the substrate with the operating zone length about $200 \mathrm{~mm}$ before condensing a metal. After the end of the experiment, the substrate has been left to cool to room temperature and wetting angles have been measured on the crystallized drops being condensed at different temperatures of the substrate. As is shown by studies performed directly inside the electron microscope, one observes no disruption of the wetting perimeter on heating the drops up to the start of their evaporation as well as on cooling them to room temperature. As the radius of the drop base remains unchanged (perhaps, due to the wetting hysteresis) and the drop keeps its spheric form, the observed changes of wetting angle values on cooling can occur only due to changes of its volume. The estimate of possible $\theta$ changes due to thermal compression of the metal and the volume jump under crystallization [8,9] gives values less than $2^{\circ}$, what is within the measurement error $\left( \pm 3^{\circ}\right)$. Therefore, one has a right to attribute the angle $\theta$ values measured on crystallized drops to the temperatures of their formation under condensation. The wetting angles have been measured from the electron micrographs of particle profiles (Fig. 1), the average $\theta$ values being found by averaging over 10-20 microparticles including the errors in wetting angle values.

\section{Results and discussion}

\subsection{Size effect on wetting}

Fig. 2 gives the measured values of wetting angles in the $\mathrm{Sn} / \mathrm{C}$ system at $T=400 \mathrm{~K}$ from which one observes the decrease of the wetting angle with the drop size decreasing for supercooled as well as for equilibrium drops [4-7]. However, the numerical values of wetting angles for drops of equal size turn out to be different and the $\theta(R)$ dependence ( $R$ is the drop radius) is shifted to the region of lesser $\theta$ values by $\Delta \theta \approx 15^{\circ}$ for supercooled drops.

One can treat these results accounting for the size dependence of the liquid phase surface energy $\sigma_{1}(R)$ and the interphase energy of the drop-substrate boundary $\sigma_{\mathrm{ul}}(R)$. Comparison with the known data on size effect on wetting at $T>T_{\mathrm{s}}$ [4-7] permits to consider the mechanism of the effect for metastable drops (at $T<T_{\mathrm{s}}$ ) to be identical to the one for equilibrium drops, i.e., to be due to the size 


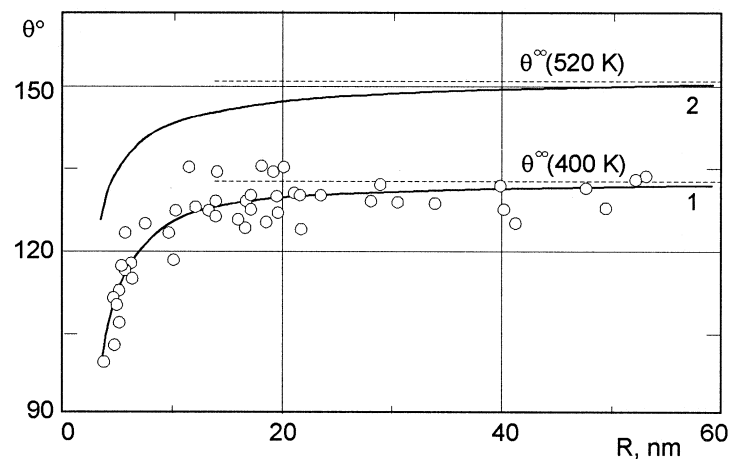

Fig. 2. Wetting angle against the supercooled $(1-T=400 \mathrm{~K})$ and equilibrium $(2-T=520[4,5])$ tin microdrop radii.

dependence of the surface energy of a drop itself and the interphase energy of the drop-substrate boundary [4-7].

In the frames of this assumption, the data may be treated according to the technique described in Refs. [6,7] using the relation describing microdrop equilibrium on a solid substrate. One can write the dependence of the wetting angle of the drop surface curvature radius $R$ including the size dependences $\sigma_{\mathrm{l}}(R)$ and $\sigma_{\mathrm{ul}}(R)$ as follows

$\cos \theta=\frac{\sigma_{\mathrm{u}}-\sigma_{\mathrm{ul}}-R \mathrm{~d} \sigma_{\mathrm{u}} / \mathrm{d} R-(r / 2) \mathrm{d} \sigma_{\mathrm{ul}} / \mathrm{d} r}{\sigma_{1}+R \mathrm{~d} \sigma_{\mathrm{l}} / \mathrm{d} R}$,

where $r=R \sin \theta$ is the drop base radius. If one applies the wellknown approximations

$\sigma_{1}=\sigma_{1}^{\infty}(1-\alpha / R)$ and $\sigma_{\mathrm{ul}}=\sigma_{\mathrm{ul}}^{\infty}(1-\beta / r)$

to the specific energies of corresponding interphase surfaces $[10,11]$, then, one can obtain for the wetting angle size dependence the expression [6,7]

$\cos \theta=\cos \theta^{\infty}-\frac{\alpha}{R}+\frac{\beta}{2 R} \frac{\sigma_{\mathrm{ul}}^{\infty}}{\sigma_{1}^{\infty}} \frac{1}{\sin \theta}$.

Here, $\alpha$ and $\beta$ are the positive parameters order of the interatomic distance determining the size dependences of specific energies of corresponding interphase boundaries, the superscript $\infty$ denoting the value for a bulk material.

One should note the difficulty in numerical treatment of measured data because of the lack of data on the surface energy of metals under large supercoolings. The temperature dependence of the surface energy for a number of metals $(\mathrm{Sn}, \mathrm{Bi}, \mathrm{In}, \mathrm{Pb}$ and $\mathrm{Ga}$ ) in a supercooled state has been measured in paper [12] with sessile drop technique. One has not observed any peculiarities on passing through the melting point on the $\sigma_{1}(T)$ dependence. Thus, for tin in the $470-540 \mathrm{~K}$ temperature range, the dependence $\sigma_{1}(T)$ is linear and permits a good approximation $\sigma_{1}(T)=\sigma_{1}\left(T_{\mathrm{s}}\right)$ $-A\left(T-T_{\mathrm{s}}\right)$, where $\sigma_{\mathrm{l}}\left(T_{\mathrm{s}}\right)=550 \mathrm{~mJ} / \mathrm{m}^{2}, A \approx 0.1 \mathrm{~mJ} / \mathrm{m}^{2}$ $\mathrm{K}$. Therefore, to determine $\sigma_{1}$ at $T<T_{\mathrm{s}}$, it is appropriate to apply the linear extrapolation of available data relating to higher temperatures [12]. Using this value $\sigma_{1}=560$ $\mathrm{mJ} / \mathrm{m}^{2}$ at $T=400 \mathrm{~K}$ and assuming the pattern of the size dependence $\theta(R)$ to persist in the supercooled state, too, i.e., taking for the parameter $\alpha$ the value corresponding to equilibrium drops $(\alpha=0.25 \mathrm{~nm}[6,7])$, one gets with the help of formula (3), the values $\beta=1.6 \mathrm{~nm}, \theta^{\infty}=134^{\circ}$ and $\sigma_{\mathrm{ul}}^{\infty}=500 \mathrm{~mJ} / \mathrm{m}^{2}$. For equilibrium drops, the corresponding parameters of the dependence $\theta(R)$ turn out to be different: $\beta=1.0 \mathrm{~nm}, \theta^{\infty}=152.7^{\circ}$ and $\sigma_{\mathrm{ul}}^{\infty}=592 \mathrm{~mJ} / \mathrm{m}^{2}$ $\left(\sigma_{1}^{\infty}=531 \mathrm{~mJ} / \mathrm{m}^{2}, \sigma_{\mathrm{u}}=120 \mathrm{~mJ} / \mathrm{m}^{2}\right)[6,7]$.

Thus, studying the size effect in wetting of supercooled vacuum condensates has discovered the dependence $\theta(R)$ at $T>T_{\mathrm{s}}$ to obey the wellknown relationships but the parameter values $\theta^{\infty}, \sigma_{1}^{\infty}, \sigma_{\mathrm{ul}}^{\infty}$ are different below and above $T_{\mathrm{s}}$. This points to the necessity of detailed studying of the temperature dependence of wetting in the range of the supercooled state.

\subsection{Temperature dependence of wetting}

Fig. 3 shows the measured wetting angles in $\mathrm{Sn} / \mathrm{C}$ and In/C systems. The temperature dependences obtained turn out to be nonmonotonous and possess the maxima at the temperatures 550 and $500 \mathrm{~K}$ for $\mathrm{Sn}$ and In, respectively. Below $T_{\mathrm{s}}$, the wetting angle decreases smoothly with lowering temperature. The decrease in $\theta$ for the systems studied amounts approximately to $25^{\circ}$ with maximum supercoolings attained $\Delta T_{\mathrm{Sn}}=160 \mathrm{~K}$ and $\Delta T_{\mathrm{In}}=100 \mathrm{~K}$. The improvement of wetting is also observed above $T_{\mathrm{s}}$ with the temperature increasing, the angle $\theta$ decreasing in the same
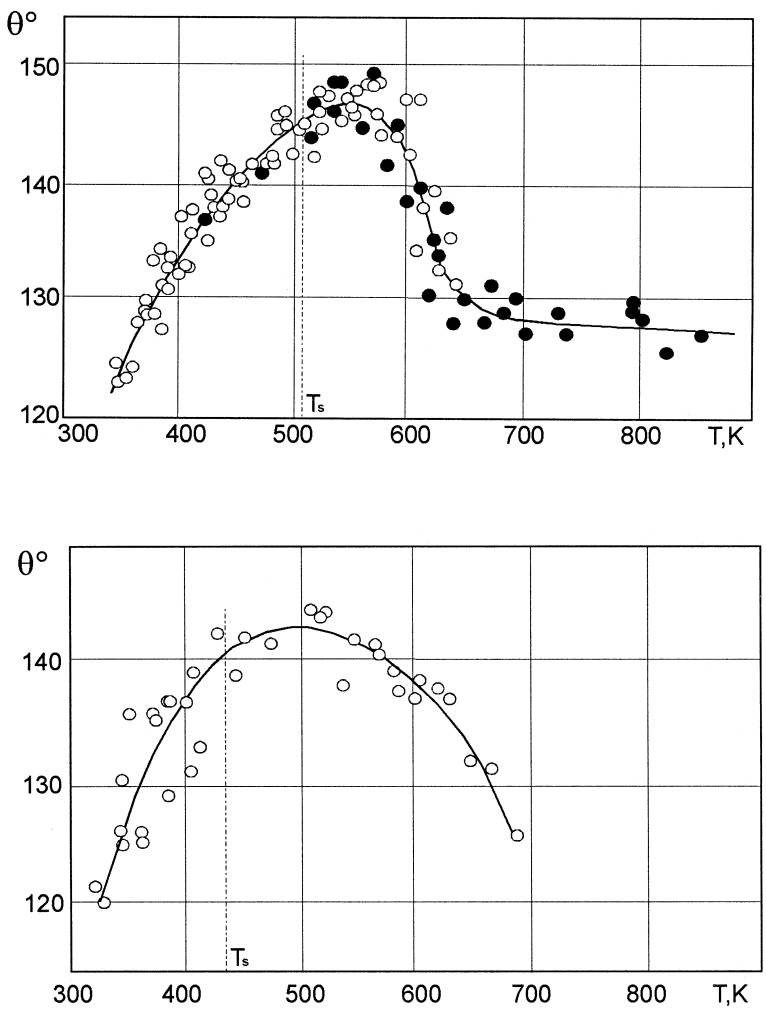

Fig. 3. Angle of wetting thick carbon films on $\mathrm{NaCl}(\circ)$ and $\mathrm{Al}_{2} \mathrm{O}_{3}(\cdot)$ by $\mathrm{Sn}$ (a) and In (b) against temperature. 
temperature range $550<T<650 \mathrm{~K}$ for indium and tin. This evidences the dependence $\theta(T)$ to be related to changes of properties of the surface under wetting within this temperature range, e.g., to the increase of the surface energy of the carbon film due to the decrease of the quantity of the adsorbed gases on its surface. That is, one should consider the system 'liquid tin-adsorbed gases layer-carbon film' and the dependence $\theta(T)$ is found to be similar to the dependence of the wetting angle on the thickness of the intermediate layer deposited on the substrate $[4,5]$. This is supported by the fact that above $700 \mathrm{~K}$ when the gas adsorption decreases considerably, the wetting angle in the $\mathrm{Sn} / \mathrm{C}$ depends on temperature linearly $\left(\mathrm{d}(\cos \theta) / \mathrm{d} T \approx-1.4 \cdot 10^{-4} 1 / \mathrm{K}\right)$, that being typical for noninteracting systems $[13,14]$ to which the contact pair noted is related.

In the range of supercooled state, the improvement of wetting evidences the essential temperature changes of the surface energy of the liquid metal or of the interphase energy of the drop-substrate boundary. One should note that surface properties of supercooled melts are poorly studied because of considerable experimental difficulties, and theoretical treatments are ambiguous. According to Ref. [15], at considerable $\Delta T$, one may expect the inversion of the temperature dependence $\sigma_{1}(T)$ due to the different temperature patterns of the volume and surface entropy. Positive $\mathrm{d} \sigma_{1} / \mathrm{d} T$ coefficients have also been observed above $T_{\mathrm{s}}$ while studying various alloys and they are due to a strong surface segregation of the substance dissolved [16]. The surface energy of supercooled metals $(\mathrm{Pb}$, $\mathrm{Sn}, \mathrm{Bi}, \mathrm{In}, \mathrm{Ga}$ ) has been measured in Ref. [12], according to which the $\sigma_{1}(T)$ pattern persists on passing through the melting point. However, these data give no answer to the question on the expected inversion of the surface energy, as in Ref. [12] the sufficiently large supercoolings have not been attained. The surface energy of the liquid phase in a supercooled state may change within the certain limits. If one assumes the interphase energy $\sigma_{\mathrm{ul}}$ to be constant or growing with the temperature decreasing, then in agreement with the Young equation, the measured data on $\theta(T)$ indicate the considerable decrease of the surface energy of the supercooled liquid metal. Thus, for tin at $T<400 \mathrm{~K}$, the value of the quantity $\sigma_{1}$ calculated for a constant adhesive tension exceeds the corresponding one for the solid metal [17-19]. Therefore, the tin crystallization at $T<400 \mathrm{~K}$ might be accompanied by the surface energy decrease that contradicts the existing theoretical and experimental data. Hence, the quantity $\sigma_{1}$ turns out to have an upper limit $\sigma_{\mathrm{s}}$ at the same temperature. Again, the $\sigma_{1}$ decrease with the temperature lowering due to the inversion should lead to the increase in wetting angles (at $\theta>90^{\circ}$ ) in contradiction to the measured data obtained. Including the above argument and the available measured data on the surface energy of a supercooled tin [12], it seems most probable to assume $\sigma_{1}(T)$ to be linear in the region of deep supercoolings. Then, the measured data on wetting in a supercooled state indicate the decrease of the interphase energy of the supercooled drop-substrate boundary with a temperature lowering. The reason of such behaviour of interphase energy is not clear yet. The change of the interphase energy $\sigma_{\mathrm{ul}}$ with the temperature lowering from $T_{\mathrm{s}}$ to the temperature of maximum supercooling when calculated with the linear extrapolation of the data of Ref. [12] on $\sigma_{1}(T)$ in the region of large supercoolings amounts to $160-180 \mathrm{~mJ} / \mathrm{m}^{2}$ or $\Delta \sigma_{\mathrm{ul}} \approx 0.3 \sigma_{\mathrm{ul}}\left(T_{\mathrm{s}}\right)$.

\section{Conclusions}

1. Application of island vacuum condensates permits efficient studies of surface properties of small liquid particles in the range of their deep supercoolings.

2. One observes the size effect on wetting being due to the decrease with size of the surface energy of particles themselves and the interphase energy of the drop-substrate boundary for supercooled tin condensates on amorphous carbon substrates as well as for the equilibrium ones.

3. We have discovered the inversion (the nonmonotonous pattern) of the temperature dependence of wetting carbon substrates by tin and indium consisting in that, the improvement of wetting is observed not only with the temperature increasing but also with the temperature decreasing in the range of the supercooled state of a liquid metal.

\section{Acknowledgements}

It is a pleasure to express my gratitude to Prof. N.T. Gladkikh for the constant attention to my work and the fruitful discussion of the results.

\section{References}

[1] N.T. Gladkikh, S.V. Dukarov, V.N. Sukhov, Z. fur Metallkunde 87 (1996) 233.

[2] S.P. Chizhik, N.T. Gladkikh, L.K. Grigoryeva, R.N. Kuklin, V.I. Larin, Zh. Eksp. Teor. Fiz. 88 (1985) 1706.

[3] S.P. Chizhik, N.T. Gladkikh, L.K. Grigoryeva, R.N Kuklin, V.I. Larin, Sov. Phys. JETP 61 (1985) 1015.

[4] S.P. Chizhik, N.T. Gladkikh, V.I. Larin, L.K. Grigoryeva, S.V. Dukarov, S.V. Stepanova, Poverkhnost (Surface) N 12 (1985) 111.

[5] S.P. Chizhik, N.T. Gladkikh, V.I. Larin, L.K. Grigoryeva, S.V. Dukarov, S.V. Stepanova, Phys. Chem. Mech. Surf. 4 (12) (1987).

[6] N.T. Gladkikh, L.K. Grigoryeva, S.V. Dukarov et al., Fiz. Tverdogo Tela 31 (1989) 13.

[7] N.T. Gladkikh, L.K. Grigoryeva, S.V. Dukarov et al., Sov. Phys. Solid State 31 (1989) 728.

[8] N.T. Gladkikh, S.P. Chizhik, V.I. Larin, L.K. Grigoryeva, S.V. Dukarov, Poverkhnost (Surface) N 11 (1985) 124.

[9] N.T. Gladkikh, S.P. Chizhik, V.I. Larin, L.K. Grigoryeva, S.V. Dukarov, Phys. Chem. Mech. Surf. 4 (11) (1987) 3465.

[10] R.C. Tolman, J. Chem. Phys. 17 (1949) 333. 
[11] W. Vogelsberger, J. Sonnerfeld, G.Z. Rudakoff, Phys. Chem. 266 (1985) 225.

[12] S.N. Zadumkin, Kh.I. Ibragimov, D.T. Ozniev, Izvestiya vuzov Tsvetnaya Metallurgiya (Non-ferrous metallurgy) N 1 (1979) 82, in Russian.

[13] S.K. Rhee, Mater. Sci. Eng. 16 (1974) 45.

[14] A.W. Adamson, Physical Chemistry of Surfaces, Wiley-Interscience, New York, 1976.
[15] C.A. Croxton, Liquid State Physics-A Statistical Mechanical Introduction, Cambridge Univ. Press, London, 1974.

[16] N. Eustathopoulos, J. Joud, Curr. Topics Mater. Sci. 4 (1980) 281.

[17] V.K. Kumikov, Kh.B. Khokonov, J. Appl. Phys. 54 (1983) 1346.

[18] N.T. Gladkikh, S.V. Dukarov, V.I. Larin, Functional Mater. 1 N 2 (1994) 49.

[19] L.Z. Mezey, J. Giber, Jpn. J. Appl. Phys. 21 (1982) 1569. 